\title{
P114 "Stifflammation" is an Essential Cause of Cardiovascular Hospitalizations in Hypertensives
}

\author{
Georgia Christopoulou*, Charalambos Vlachopoulos, Lambros Korogiannis, Dimitrios Terentes-Printzios, \\ Evangelia Sigala, Iosif Koutagiar, Vasiliki Gardikioti, Dimitrios Tousoulis
}

1st Department of Cardiology, Hippokration Hospital, University of Athens, Greece

\section{ABSTRACT}

Purpose/Background/Objectives: Hypertension is associated with increased cardiovascular risk, inflammation and arterial stiffness. We sought to investigate the role of inflammation and arterial stiffness in the prognosis of cardiovascular hospitalization in hypertensives patients over an extensive follow-up.

Methods: One hundred and seventy-three patients (mean age $52.5 \pm 13.2$ years, 57\% males) untreated hypertensives at baseline without cardiovascular disease, were included in the study. Arterial stiffness was assessed with carotid-femoral pulse wave velocity (PWV). High-sensitivity C-reactive protein (hsCRP) was measured in venous blood samples.

Results: During $13.6 \pm 0.4$ years of follow-up, forty-four patients (25.4\%) patients were admitted in hospital due to cardiovascular causes. In multivariable logistic regression analysis, only higher hsCRP (Odds Ratio $[\mathrm{OR}]=3.34,95 \%$ Confidence intervals $[\mathrm{CI}]: 1.22-9.51, p=0.020)$ and increased PWV $(\mathrm{OR}=1.48,95 \%$ Confidence intervals [CI]: 1.03-2.12, $p=0.036)$ were associated with higher risk of cardiovascular hospitalizations, which was independent of age, gender, systolic blood pressure, left ventricular mass index and presence of diabetes. In further analysis, receiver operating characteristic (ROC) curves were generated to evaluate the ability of hsCRP and PWV to discriminate subjects with cardiovascular hospitalization. The area under the curve (AUC) and 95\% CIs of the ROC curves were AUC $=0.69$ (95\% CI: 0.59-0.78, $p<0.001$ ) for hsCRP and AUC $=0.74$ (95\% CI: $0.65-0.83, p<0.001)$ for PWV (Figure 1).

Conclusion: Our study shows the independent complimentary prognostic role of inflammation and arterial stiffness in the prognosis of hypertensives even in studies with extensive follow-up.

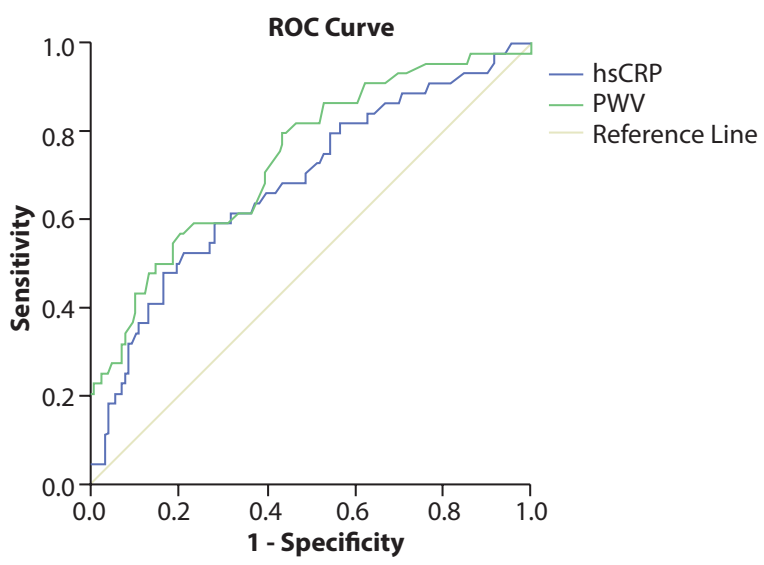

Figure 1 Diagonal segments are produced by ties.

(c) 2019 Association for Research into Arterial Structure and Physiology. Publishing services by Atlantis Press International B.V. This is an open access article distributed under the CC BY-NC 4.0 license (http://creativecommons.org/licenses/by-nc/4.0/). 